\section{A Simple Strategy to Communicate about Climate Attribution}

Rachel Dryden and M. Granger Morgan

\begin{abstract}
Hurricane Harvey and other recent weather extremes stimulated extensive public discourse about the role of anthropogenic climate change in amplifying, or otherwise modifying, such events. In tandem, the scientific community has made considerable progress on statistical "climate attribution." However, explaining these statistical methods to the public has posed challenges. Using appropriately designed "spinner boards," we find that even members of the general public who do not understand the difference between weather and climate are readily able to understand basic concepts of attribution and explain those concepts to others. This includes both understanding and explaining the way in which the probability of an extreme weather event may increase as a result of climate change and explaining how the intensity of hurricanes can be increased. If properly developed and used by TV weather forecasters and news reporters, this method holds the potential to significantly improve public understanding of climate attribution.
\end{abstract}


AFFILIATIONS: Dryden-Department of Engineering and Public Policy, Carnegie Mellon University, and RAND Corporation, Pittsburgh, Pennsylvania; Morgan—Department of Engineering and Public Policy, Carnegie Mellon University, Pittsburgh, Pennsylvania

A Imost every time there is another extreme weather event there is public discussion about whether it was caused by climate change. While there are now growing numbers of events that likely would not have occurred without anthropogenic climate change (Herring et al. 2019), in many cases, extreme events have simply become more likely because of the influence of a changing climate (Bindoff et al. 2013; Knutson et al. 2019; Bellprat et al. 2019; Reed et al. 2020). The process of making such statistical associations is now widely referred to as "climate attribution." As part of a series of studies to explore public perceptions and understanding of issues surrounding climate attribution, we developed a simple, explanatory strategy based on the use of "spinner boards," which appears to be remarkably successful. The spinner board results are part of a face-to-face interview study, which we used to lay the foundation for a larger study of when and to what extent laypeople attribute hurricanes to climate change using signal detection theory (Dryden et al. 2020). Here, we only provide a brief description of the study design in order to provide context for the spinner board results. None of the 28 respondents had technical backgrounds. As in previous studies with laypeople (Reynolds et al. 2010), they displayed limited understanding of climate science. Sixteen of the respondents believed weather and climate to be essentially the same thing or did not know the difference. Additional details of both this study and the larger study using signal detection theory can be found in Dryden Steratore (2019).

A standard way of communicating about the return period of rare events is to talk in terms of an N-year event. For example, USGS speaks of 100-year floods and produces maps of the 100-year floodplains. In such a case, the annual event probability $\mathrm{P}(E)$ is $0.01 .{ }^{1} \mathrm{We}$ sought to explain such processes to laypeople by using spinner boards (Fig. 1a), in which the odds of the event occurring are equal to the chance that a spinning pointer will land on the narrow colored segment of the board whose width is $\mathrm{P}(E) \times 360^{\circ} .^{2}$ We explained the fact that a "100-year flood" could happen two years in a row by noting that, while it was most unlikely, there is a slim chance that the spinning pointer could land twice in a row on the narrow red slice (which, in the 1-in-100 case, would be only $0.01 \times 360=3.6^{\circ}$ wide). Of course, if the value of $\mathrm{P}(E)$ is more than one expected event per year, then a briefer period should be used for the denominator (i.e., events per month or even

There is, of course, no reason why the formulation needs to be made in terms of events per year. Any period of time can be used. However, for rare events, a year (or longer) is an interval most people easily understand.

² MIT’s Global Change group has used very different types of spinner boards to describe the uncertainty of future climate predictions with and without policy intervention (Sokolov et al. 2009). some shorter period).

Once a respondent in the face-to-face interview had played with the board for a few moments, we asked: "If climate change makes it more likely that an extreme event will occur, how would the spinner board change, and what would this difference mean?" In face-to-face interviews conducted with 28 lay respondents through Carnegie Mellon University's Center for Behavioral and Decision Research, every single respondent correctly explained that the effect of climate change would make the colored segment get a bit wider 
(i.e., without seeing Fig. 1b). We next asked respondents to imagine that they were going to use the two spinner boards in Fig. 1 to explain the effect of climate change on the likelihood of an extreme event to a neighbor or friend. Once again, every single respondent provided a correct explanation.

Turning our focus to hurricanes, we showed respondents the spinner board shown in Fig. 2a, in which we divided a spinner in proportion to the occurrence of the 1-5 Saffir-Simpson hurricane intensity values. Again, we asked respondents to explain the spinner for the case of a specific storm event, which they did correctly. Then we asked, "Suppose that climate change makes it more likely that hurricanes will become more intense. How would this spinner board change?” Again, without seeing Fig. 2b, every respondent correctly explained that the width of the colored sections for the higher Saffir-Simpson hurricane intensity values (i.e., categories 3-5) would get wider. Finally, we gave them the two spinners in Fig. 2 and asked them to provide an explanation to a neighbor or friend. All respondents provided a correct explanation.
A.
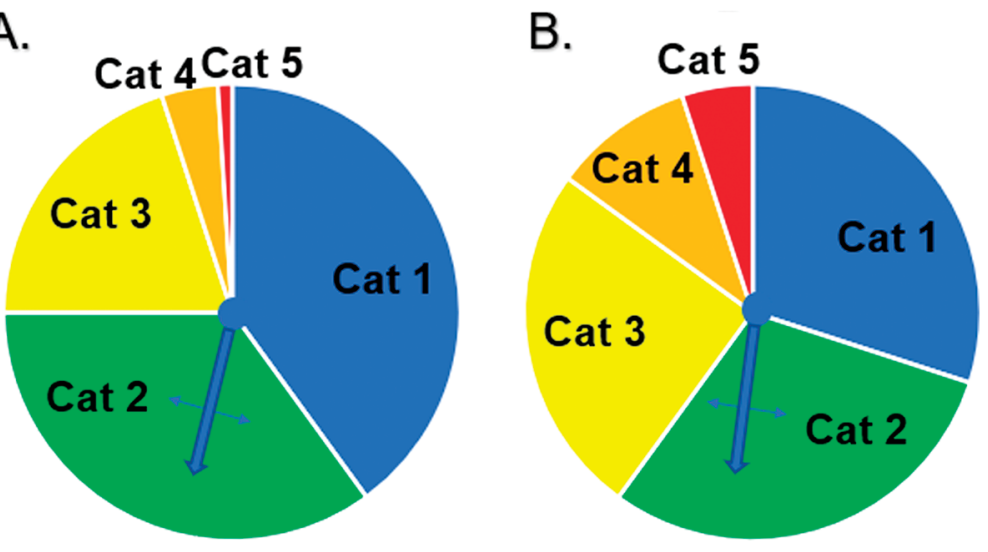

Fig. 2. (a) Basic spinner board used to explain that the distribution of peak hurricane strengths could change in the future. (b) Modified spinner board showing how climate change might increase the probability that a storm would be more intense. As in Fig. 1, in our study, all respondents were able to describe the modified board before seeing it, and then when given the second board, correctly used the pair of boards to explain the effect of climate change on the intensity of a hurricane to a friend or neighbor This figure shows the spinner boards used in this study. It is not intended to represent any actual expected change in the frequency of any specific extreme events.

\section{Broader context of the study}

On average, the face-to-face interviews reported here lasted about $30 \mathrm{~min}$. Participants were compensated $\$ 5.00$ for their time. The ages of respondents ranged from 18 to 76 years old (mean = 31.0 , median $=26$ ). The average age of this convenience sample was younger than the U.S. populations (U.S. mean = 41, U.S. median = 37). This sample was better educated than the U.S. population: all 28 had finished high school [U.S. = 88\%], 17 (61\%) had completed college [U.S. $_{\text {some }}=59 \%$; U.S. $_{\text {all }}=33 \%$ ], and $12(43 \%)$ had completed graduate training [U.S. $=12 \%$ ]. Of the total sample, 20 (71\%) identified as liberal [U.S. $=47 \%$ ] and 15 (54\%) were female [U.S. $=51 \%$ ].

The interview protocol adopted wording from our previous studies on public understanding of climate change (Dryden et al. 2018; Reynolds 
et al. 2010; Bostrom et al. 1994; Read et al. 1994). Additional questions were pretested in a set of pilot interviews. The interview protocol consisted of three sections: 1) trends in the weather; 2) meaning, examples of, and perceptions of the causes of extreme weather events; and 3) questions surrounding climate attribution, including the use of spinner boards. All interviews were recorded, and the audio recordings were transcribed verbatim and coded in ATLAS.ti based on thematic patterns. Some broad themes we examined included climate change and extreme weather beliefs, the presence of pro-environmental attitudes, and understanding of causality and probabilities. For example, when asked to provide examples of extreme weather events, hurricanes were the most frequently cited example (86\%), followed by heavy rainfall (68\%) and flooding (64\%). When asked to list extreme events, nearly all participants also cited events not driven by weather patterns, like earthquakes and volcanic eruptions. When asked what changes in weather could happen because of $\mathrm{CO}_{2}$ being added to the atmosphere, all participants cited more extreme weather and global warming/warmer temperatures. Since the sample size in this study was modest, we do not report details on the other parts of the study here. Interested readers can find those results, along with the full interview protocol and demographic details, in Dryden Steratore (2019). In most cases, results in this study were similar to findings from other studies of public understanding of climate change that we and others have conducted (Dryden et al. 2018; Reynolds et al. 2010; Bostrom et al. 1994; Read et al. 1994).

\section{Conclusions}

The science of climate attribution has made enormous progress in recent years. While discussions of climate attribution of extreme events can be found in the literature (Herring et al. 2016) and in the media (Guskin and Dennis 2017), public understanding of the issue of attribution, and the interpretation of non-stationary events, has not kept pace.

In the face of limited public understanding of climate science, the use of spinner boards appears to be an easily understood and highly effective strategy to explain issues of climate attribution to the general public. Spinner boards could be readily adapted for use by TV weather forecasters and in news coverage of hurricanes and other extreme weather events. In the past, many TV weather forecasters have been reluctant to mention climate change when talking about extreme events, although their views and acceptance are evolving (Maibach et al. 2017). Despite this, the (basically) correct understanding of attribution that we observed when explained in terms of spinner boards may provide weather forecasters and news reporters with a vehicle to provide compelling visual explanations, as well as a basis for arguing that employing such displays can be readily justified.

Acknowledgments. This work has been supported by the Center for Climate and Energy Decision Making (CEDM) through a cooperative agreement between the National Science Foundation and Carnegie Mellon University (SES-0949710) and by the Hamerschlag Chair and other academic funds from Carnegie Mellon University. 


\section{REFERENCES}

Bellprat, O., V. Guemas, F. Doblas-Reyes, and M. G. Donat, 2019: Towards reliable extreme weather and climate event attribution. Nat. Commun., 10, 1732, https://doi.org/10.1038 Is41467-019-09729-2.

Bindoff, N. L., and Coauthors, 2013: Detection and attribution of climate change: From global to regional. Climate Change 2013: The Physical Science Basis, T. F. Stocker et al., Eds., Cambridge University Press, 867-952.

Bostrom, A., M. G. Morgan, B. Fischhoff, and D. Read, 1994: What do people know about global climate change? 1. Mental models. Risk Anal., 14, 959-970, https://doi .org/10.1111/j.1539-6924.1994.tb00065.x.

Dryden, R., M. G. Morgan, A. Bostrom, and W. Bruine de Bruin, 2018: Public perceptions of how long air pollution and carbon dioxide remain in the atmosphere. Risk Anal., 38, 525-534, https://doi.org/10.1111/risa.12856.

_ _ _ and S. Broomell, 2020: Lay detection of unusual patterns in the frequency of hurricanes. Wea. Climate Soc., in press. https://doi.org/10.1175/WCAS-D-19-0132.1.

Dryden Steratore, R. L., 2019: Public understanding of climate science, extreme weather and climate attribution. Ph.D. dissertation, Carnegie Mellon University, 156 pp.

Guskin, E., and B. Dennis, 2017: Majority of Americans now say climate change makes hurricanes more intense. Washington Post, 28 September, www.washingtonpost.com/news/energy -environment/wp/2017/09/28/majority-of-americans-now -say-climate-change-makes-hurricanes-more-intense/?utm _term $=.98 \mathrm{c} 8 \mathrm{cdf} 1 \mathrm{e} 453$.

Herring, S. C., A. Hoell, M. P. Hoerling, J. P. Kossin, C. J. Schreck III, and P. A. Stott, 2016: Introduction to Explaining Extreme Events of 2015 from a Climate Perspective [in "Explaining
Extreme Events of 2015 from a Climate Perspective"]. Bull. Amer. Meteor. Soc., 97 (12), S1-S3, https://doi.org/10.1175 /BAMS-D-16-0313.1.

—,$- ~-, ~ N$. Christidis, and P.A. Stott, 2019: Introduction to Explaining Extreme Events of 2017 from a Climate Perspective [in "Explaining Extreme Events of 2017 from a Climate Perspective"]. Bull. Amer. Meteor. Soc., 100 (1), S1S4, https://doi.org/10.1175/BAMS-D-18-0307.1.

Knutson, T., and Coauthors, 2019: Tropical cyclones and climate change assessment: Part I: Detection and attribution. Bull. Amer. Meteor. Soc., 100, 1987-2007, https://doi.org/10.1175 /BAMS-D-18-0189.1.

Maibach, E., and Coauthors, 2017:TV weathercasters' views of climate change appear to be rapidly evolving. Bull.Amer. Meteor. Soc., 98, 2061-2064, https://doi.org/10.1175/BAMS-D-15-00206.1.

Read, D., A. Bostrom, M. G. Morgan, B. Fischhoff, and T. Smuts, 1994: What do people know about global climate change? 2. Survey studies of educated laypeople. Risk Anal., 14, 971982, https://doi.org/10.1111/j.1539-6924.1994.tb00066.x.

Reed, K. A., A. M. Stansfield, M. F. Wehner, and C. M. Zarzycki, 2020: Forecasted attribution of the human influence on Hurricane Florence. Sci. Adv., 6, eaaw9253, https://doi.org/10.1126 ISCIADV.AAW9253.

Reynolds, T. W., A. Bostrom, D. Read, and M. G. Morgan, 2010: Now what do people know about global climate change? Survey studies of educated laypeople. Risk Anal., 30, 1520-1538, https://doi.org/10.1111/j.1539-6924.2010.01448.x.

Sokolov, A. P., and Coauthors, 2009: Probabilistic forecast for twenty-first-century climate based on uncertainties in emissions (without policy) and climate parameters. J. Climate, 22, 5175-5204, https://doi.org/10.1175/2009JCLI2863.1 